\title{
Design of a Channel-Aware OFDM Transceiver
}

\author{
Ji-Woong Choi, Member, IEEE, and Yong-Hwan Lee, Member, IEEE
}

\begin{abstract}
The transmission performance of an OFDM system can significantly be improved by exploiting the channel characteristics. In this paper, we consider the design of a channel-aware OFDM transceiver whose parameters are adjusted in response to the change of channel condition. To this end, we first estimate the channel state information (CSI), such as the signal to interference power ratio, low order moments of Doppler spectrum and powerdelay profile of the channel. The proposed CSI estimator can estimate these CSI parameters altogether in a unique manner by exploiting the autocorrelation properties of the channel impulse response (CIR). Then, we design a CIR estimator and adaptive OFDM modulator that adjust their parameters according to the estimated CSI. Finally, we verify the performance of the proposed OFDM transceiver by computer simulation
\end{abstract}

Index Terms-Channel-aware OFDM transceiver, channel state information, channel impulse response, adaptive modulation with coding.

\section{INTRODUCTION}

$\mathbf{R}$ ECENTLY, orthogonal frequency division multiplexing (OFDM) has attracted much attention as an effective modulation technique for next generation wireless access systems. In practice, OFDM based transmission technologies have been employed in the European terrestrial digital video broadcasting system and wireless local area networks [1,2]. The standardization body is also considering the OFDM or its variation as a primary candidate for the air interface of next generation wireless systems $[3,4]$.

In order to maximize the data throughput and performance, the OFDM system needs to adjust its transceiver parameters in response to the change of channel condition. The potentials of adaptive techniques were recognized long time ago [5], but they did not receive much interest at that time mainly due to the implementation complexity and lack of good channel estimation techniques. In the last decade, however, advances in fast and reconfigurable transceiver technologies have renewed the interest in the use of adaptive systems such as adaptive modulation with coding (AMC), adaptive antenna and equalization techniques.

Accurate channel state information (CSI) is indispensable for the employment of adaptive channel-aware techniques in

Manuscript received August 31, 2005; revised September 16, 2006; accepted December 26, 2006. The associate editor coordinating the review of this paper and approving it for publication was V. Lau. This work was presented in part at the IEEE International Conference on Communications, May 2005. This work was in part supported by the Ministry of Information \& Communications, Korea, under the Information Technology Research Center (ITRC) Support Program.

J.-W. Choi was with the School of Electrical Engineering and Computer Science and INMC, Seoul National University, Seoul 151-744, Korea. He is now with the Department of Electrical Engineering, Stanford University, Stanford, CA 94305 (e-mail: jwchoi@ stanford.edu).

Y.-H. Lee is with the School of Electrical Engineering and Computer Science and INMC, Seoul National University, Seoul 151-744, Korea (e-mail: ylee@snu.ac.kr).

Digital Object Identifier 10.1109/TWC.2007.05642. the OFDM system. A number of studies have investigated on the estimation of CSI parameters, such as the average signal to interference power ratio (SIR) and the maximum Doppler frequency [6,7]. However, these CSI parameters may not be sufficient for the employment of advanced channelaware techniques in the OFDM. For example, the transceiver performance can significantly be affected by the shape of the Doppler spectrum. The Doppler spectrum can have a different shape depending on the existence of the line-of-sight (LOS) component or scatterers' distribution even when the maximum Doppler frequency is unchanged [8]. Thus, the moments of the Doppler spectrum need to be considered as the major CSI parameter in the time domain since they can manifest the shape of the Doppler spectrum [9]. In addition, the moments of the power-delay profile can describe the channel characteristics in the frequency domain better than the maximum delay spread. Thus, we consider the use of low order moments of the Doppler spectrum and power-delay profile as the CSI as well as the average SIR. To the best of authors' knowledge, no result has been reported on the estimation of these CSI parameters using a unified scheme. We design such a CSI estimator by exploiting the autocorrelation properties of the channel impulse response (CIR). Then, we consider the use of the estimated CSI for the design of channel-aware OFDM transceivers.

We first consider the use of CSI for the estimation of CIR. An optimum channel estimation filter (CEF) can be realized using complex-coefficient Wiener filters that minimize the mean squared error (MSE) of the CIR estimation [15]. However, it may not be applicable to the real world mainly due to huge implementation complexity. In practice, the CEF is often realized using simple interpolation filters such as linear, Lagrange and spline interpolation schemes [16]. However, the use of simple CEFs with fixed coefficient may not provide desired performance under certain channel condition. Thus, it is desirable to employ a CEF whose parameters are adjusted according to the channel condition with an acceptable complexity. A previous work considered the selection of a CEF among four CEFs according to the amplitude and autocorrelation of pilot symbol [17]. However, it cannot provide optimum performance because four CEFs were designed in a heuristic manner. To alleviate the implementation problem, we consider the use of a two-dimensional (dim) cascaded type CEF that comprises a linear interpolator and a moving average (MA) filter. The tap size of the MA finite impulse response (FIR) filter is adjusted based on the estimated CSI so as to minimize the MSE of the CIR estimation [10]. We also consider the use of a delay-shifting process in the frequency domain to avoid performance degradation due to the use of a real-coefficient CEF.

We also consider the use of the CSI for the AMC. The 
AMC mode is usually determined by comparing the SIR with a threshold. Applying different AMC modes to each subcarrier with frequent update, it can be possible to obtain near optimum performance [19]. However, it may suffer from so-called feedback signaling burden. In this paper, we consider a low feedback scheme where all the subcarriers use the same mode which is updated once in a while for easy implementation. In this case, it may be effective to use a fixed SIR threshold when the CIR is little changed within a transmission block $[23,24]$. However, it may be desirable to adjust the SIR threshold for the exploitation of channel diversity in fast fading and/or large frequency selective fading environments $[11,26]$. The SIR threshold can be optimized by analyzing the packet error rate (PER) performance. However, it may involve practical difficulty due to the effect of channel coding and correlation between the adjacent symbols. As an alternative, the SIR threshold can be adjusted based on the history of ARQ response [26], which takes a long time for convergence. In this paper, we propose to adjust the SIR threshold according to the rms value of the channel spectrum obtained from the proposed CSI estimator.

Following this Introduction, the system and channel model are described in Section II. The proposed OFDM transceiver structure is described in Section III, where a unified CSI estimator is designed after the CSI parameters are defined. Then, an OFDM transceiver is designed, that utilizes the estimated CSI for the improvement of CIR estimation and AMC. The performance of the proposed scheme is verified by computer simulation in Section IV. Finally, conclusions are summarized in Section V.

\section{SYSTEM AND CHANNEL MODEL}

Consider an OFDM transmitter where $K$ subcarrier symbols, $X[n, k], k=0,1,2, \ldots, K-1$, are converted into a time domain signal at the $n$-th symbol time by the inverse fast Fourier transform (FFT) process. The data symbols generated by a block-by-block basis comprise data bits after channel coding, interleaving and QAM-type bit interleaved coded modulation (BICM). The pilot symbols are inserted in a rectangular pattern whose spacing is $d_{t}$ and $d_{f}$ in the time and frequency domain, respectively. Before the transmission, a cyclic prefix (CP) is inserted to preserve the orthogonality between the subcarriers and to eliminate intersymbol interference.

Assume that the signal is transmitted over a wireless channel whose impulse response is represented as

$$
h(t, \tau)=\sum_{l=0}^{L-1} h_{l}(t) \delta\left(\tau-\tau_{l}\right)
$$

where $L$ is the number of multipaths unknown to the receiver, $\delta(\cdot)$ is Kronecker delta function, and $\tau_{l}$ and $h_{l}(t)$ are the delay and complex-valued CIR of the $l$-th path at time $t$, respectively. Here, $\left\{h_{l}(t)\right\}$ is modelled as an independent complex Gaussian process having the same normalized correlation function in the time domain and different average power $[12,15]$

$$
\begin{aligned}
r_{l}(\Delta t) & =E\left\{h_{l}(t+\Delta t) h_{l}^{*}(t)\right\} \\
& =\sigma_{l}^{2} r_{t}(\Delta t)
\end{aligned}
$$

where $E\{X\}$ denotes the expectation of $X$, the superscript * denotes complex conjugate and $\sigma_{l}^{2}$ denotes the average power of the $l$-th path. Let $H(t, f)$ be the frequency response of the CIR at time $t$ defined as

$$
H(t, f)=\int_{-\infty}^{\infty} h(t, \tau) \mathrm{e}^{-j 2 \pi f \tau} d \tau
$$

Assuming the use of normalized average signal power (i.e., $\left.\sigma_{s}^{2}=\sum_{l=0}^{L-1} \sigma_{l}^{2}=1\right)$, it can be shown that $[12,15]$

$$
\begin{aligned}
r_{H}(\Delta t, \Delta f) & =E\left\{H(t+\Delta t, f+\Delta f) H^{*}(t, f)\right\} \\
& =r_{t}(\Delta t) r_{f}(\Delta f)
\end{aligned}
$$

where $r_{f}(\Delta f)=\sum_{l=0}^{L-1} \sigma_{l}^{2} \mathrm{e}^{-j 2 \pi \Delta f \tau_{l}}$. The correlation function of an OFDM symbol with symbol time $T_{s}$ and subcarrier spacing $\Delta f_{c}$ can be represented in a discrete form as

$$
r_{H}[n, k]=r_{t}[n] r_{f}[k]
$$

where $r_{t}[n]=r_{t}\left(n T_{s}\right)$ and $r_{f}[k]=r_{f}\left(k \Delta f_{c}\right)$. The spectrum of the channel correlation can be represented using 2-dim discrete Fourier transform (DFT) as

$$
\begin{aligned}
S_{H}\left(w_{1}, w_{2}\right) & =\sum_{n=-\infty}^{\infty} \sum_{k=-\infty}^{\infty} r_{H}[n, k] e^{-j\left(w_{1} n+w_{2} k\right)} \\
& =\sum_{\substack{n=-\infty \\
n}}^{\infty} r_{t}[n] e^{-j w_{1} n} \sum_{k=-\infty}^{\infty} r_{f}[k] e^{-j w_{2} k} \\
& =S_{H_{1}}\left(w_{1}\right) S_{H_{2}}\left(w_{2}\right)
\end{aligned}
$$

where $S_{H_{1}}\left(w_{1}\right)$ and $S_{H_{2}}\left(w_{2}\right)$ are the DFT of $r_{t}[n]$ and $r_{f}[k]$, respectively.

The CP is removed before the FFT process in the receiver. Assuming ideal synchronization in the receiver, the received symbol of the $k$-th subcarrier at the $n$-th symbol time can be represented by

$$
Y[n, k]=X[n, k] H[n, k]+Z[n, k]
$$

where $H[n, k]$ is the frequency response of the CIR at the $k$-th subcarrier and the $n$-th symbol time, and $Z[n, k]$ is the background noise plus interference term, which can be approximated as zero mean additive white Gaussian noise (AWGN) with variance $\sigma_{Z}^{2}$. We neglect the effect of intercarrier interference assuming that the symbol duration $T_{s}$ is short enough to neglect the CIR variation during the symbol time. The CSI and CIR can be estimated using the received pilot symbols. The data symbols after the equalization based on the estimated CIR are used for the generation of a soft metric through BICM demapping. Finally, the desired data bits are obtained after deinterleaving and channel decoding process.

Characteristics of the channel can be described in terms of the autocorrelation function or its Fourier transform [13]. The time-domain characteristics of a channel can be known from the Doppler spectrum which is the Fourier transform of the autocorrelation function $r_{t}(\Delta t)$. In a Rayleigh fading channel with uniformly distributed scatterers (e.g., classic or Jakes' spectrum), the Doppler spectrum has a distribution uniquely determined by the maximum Doppler frequency $f_{d}$ [14]. However, $f_{d}$ may not exactly represent the characteristics 
of other channels such as Ricean or non-Jakes' Rayleigh fading channels whose Doppler spectrum shapes are different at the same $f_{d}$. Thus, it may need to consider the distribution of the Doppler spectrum for channel adaptive processes, in addition to $f_{d}$.

The Doppler spectrum can precisely be described in terms of its moments [9]. In practice, the time-domain characteristics of a channel can be approximated by low order moments of the Doppler spectrum. Similarly, the frequency-domain characteristics of a channel can be described by low order moments of the power-delay profile. In addition to information on the channel variation in the time and frequency domain, it may be desirable to use the average SIR information to figure out the average strength of the channel.

A few studies have been reported on the moment estimation of Doppler spectrum [7,13]. The use of the second-order moment was considered for the estimation of $f_{d}$ [7]. The scheme in [13] may not easily be realizable due to involved calculation of continuous derivatives. Thus, it is desirable to devise a scheme that can estimate high order moments of the Doppler spectrum with an affordable complexity. It is also necessary to estimate the moments of the power-delay profile. A number of studies have been reported on the measurement of the average SIR [27-29]. Consequently, the aforementioned CSI parameters can be measured by their own estimators independently, increasing the overall implementation complexity. We devise a single unified scheme that can estimate the moments of the Doppler spectrum and power-delay profile as well as the SIR altogether.

\section{Proposed OFDM Transceiver}

We consider the design of an adaptive OFDM transceiver whose block diagram is depicted in Fig. 1, where a unified CSI estimator is designed for the purpose of improved AMC and CIR estimation. At the receiver, the proposed CSI estimator calculates the average SIR and low-order moments of the channel spectrum by exploiting the autocorrelation properties of the received pilot symbols. These CSI parameters are sent to both adaptive schemes for the adaptation of the CEF in the CIR estimator and the SIR threshold in the AMC scheme. The proposed CIR estimator adjusts the CEF parameters based on the estimated CSI. The first- and second-order moment of the channel spectrum are used to adjust the SIR threshold for the AMC. Comparing the SIR threshold with the SIR measured in the CSI estimator during current correlation period, the AMC mode applied to all the subcarriers is determined at the receiver and informed to the transmitter through a feedback channel. Since these parameters are updated for each correlation period comprising tens to thousands of data blocks, the amount of additional feedback signaling burden is marginal.

\section{A. CSI estimator}

The autocorrelation function in the time and frequency domain contains information on the moments of Doppler spectrum and power-delay profile, respectively. The normalized autocorrelation function in the time domain can be represented

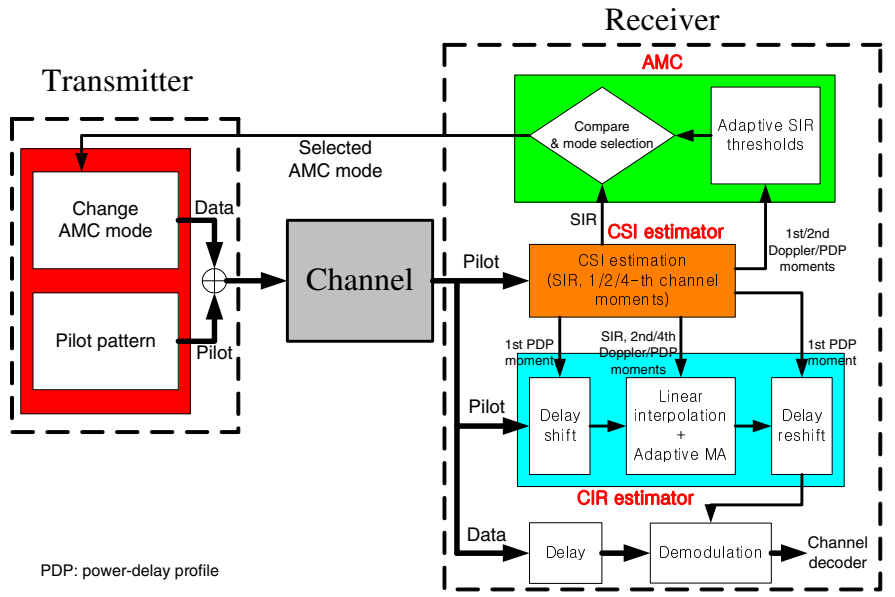

Fig. 1. Block diagram of the proposed OFDM transceiver.

as

$$
\begin{aligned}
\hat{r}_{H}[\Delta n, 0] & =\frac{E\left\{\tilde{H}[n, k] \tilde{H}^{*}[n+\Delta n, k]\right\}}{E\left\{|\tilde{H}[n, k]|^{2}\right\}} \\
& =\frac{r_{H}[\Delta n, 0]}{r_{H}[0,0]+\sigma_{z}^{2}} \\
& =\frac{r_{t}[\Delta n]}{1+\bar{\gamma}^{-1}}
\end{aligned}
$$

where $\tilde{H}[n, k](=Y[n, k] / X[n, k])$ is the instantaneous CIR estimated at the $n$-th symbol time and the $k$-th subcarrier, and $\bar{\gamma}$ denotes the average SIR of the received symbol (i.e., $E\left\{|X[n, k]|^{2}\right\} / \sigma_{z}^{2}$ ). Since $r_{t}[\Delta n]$ can be approximated using a Taylor series expansion as

$$
r_{t}[\Delta n] \approx \sum_{k=0}^{W_{t}-1} c_{k}(\Delta n)^{k}
$$

where

$$
c_{k}=\left.\frac{1}{k !} \frac{\partial^{k} r_{t}[\Delta n]}{\partial(\Delta n)^{k}}\right|_{\Delta n=0} .
$$

Thus, (8) can be rewritten as

$$
\hat{r}_{H}[\Delta n, 0] \approx \sum_{k=0}^{W_{t}-1} c_{k}^{\prime}(\Delta n)^{k}
$$

where

$$
c_{k}^{\prime}=\left(1+\bar{\gamma}^{-1}\right)^{-1} c_{k} .
$$

Letting $\bar{w}_{1}^{(k)}$ be the $k$-th order moment of Doppler spectrum defined as

$$
\bar{w}_{1}^{(k)}=\frac{1}{2 \pi} \int_{-\pi}^{\pi} w^{k} S_{H_{1}}(w) d w,
$$

it can be shown from the inverse DFT of $r_{t}[\Delta n]$ that

$$
\begin{aligned}
c_{k} & =\left.\frac{1}{k !} \frac{\partial^{k}}{\partial(\Delta n)^{k}}\left(\frac{1}{2 \pi} \int_{-\pi}^{\pi} S_{H_{1}}(w) e^{j w \Delta n} d w\right)\right|_{\Delta n=0} \\
& =\frac{j^{k}}{k !} \bar{w}_{1}^{(k)} .
\end{aligned}
$$


Letting $W_{t}=2 W$, (11) can be rewritten as

$$
\begin{aligned}
& \hat{r}_{H}[\Delta n, 0] \approx \sum_{k=0}^{2 W-1} c_{k}^{\prime}(\Delta n)^{k} \\
& =\frac{1}{1+\bar{\gamma}^{-1}} \sum_{k=0}^{2 W-1} \frac{j^{k}}{k !} \bar{w}_{1}^{(k)}(\Delta n)^{k} \\
& =\frac{1}{1+\bar{\gamma}^{-1}}\left(\sum_{k=0}^{W-1} \frac{(-1)^{k}}{(2 k) !} \bar{w}_{1}^{(2 k)}(\Delta n)^{2 k}\right. \\
& \left.+j \sum_{k=0}^{W-1} \frac{(-1)^{k}}{(2 k+1) !} \bar{w}_{1}^{(2 k+1)}(\Delta n)^{2 k+1}\right) .
\end{aligned}
$$

Note that the real and imaginary part of $\hat{r}_{H}[\Delta n, 0]$ correspond to the even and odd order moments, respectively.

Assuming that the channel is an ergodic process, $\hat{r}_{H}[\Delta n, 0]$ can further be approximated as

$$
\hat{r}_{H}[\Delta n, 0] \approx \frac{\sum_{k=0}^{K-1} \sum_{n=n_{i}}^{n_{i}+n_{s}} \tilde{H}[n, k] \tilde{H}^{*}[n+\Delta n, k]}{\sum_{k=0}^{K-1} \sum_{n=n_{i}}^{n_{i}+n_{s}} E\left\{|\tilde{H}[n, k]|^{2}\right\}}
$$

where $n_{i}$ denotes the initial symbol index and $n_{s}$ is the number of symbols for the autocorrelation in the time domain. During each correlation period, the normalized autocorrelation (16) is calculated at the CSI estimator for $W$ intervals corresponding to $\Delta n=n_{1}, n_{2}, \cdots$, and $n_{W}$, where $n_{k}=k d_{t}$. Then, the coefficients $\left\{c_{k}^{\prime}\right\}$ in (11) can be calculated from $W$ autocorrelation samples $\left\{\hat{r}_{H}[\Delta n, 0]\right\}$ using a least square curve fitting method. Thus, the moments of the Doppler spectrum can be calculated by (14), where the average SIR $\bar{\gamma}$ can be estimated by (12) with $k=0$. Note that $c_{0}=1$ due to the normalization. Thus, all the required CSI including the moments of the Doppler spectrum and the average SIR can be obtained in a unique manner. Similarly, the moments of the power-delay profile can be estimated using the normalized autocorrelation function of the received symbols in the frequency domain.

\section{B. CIR estimator}

We consider the use of a cascaded-type CEF [10], where the received signal is first interpolated using a linear interpolator to provide the instantaneous CIR and then low-pass filtered by an MA FIR filter to suppress the noise. A two-dim MA FIR CEF can analytically be designed by minimizing the MSE of the estimated CIR. The optimum tap size of the MA FIR CEF can be determined as a function of the moments of the channel spectrum and the average SIR as follows.

The CIR at the instant of pilot symbol transmission can be estimated as

$$
\begin{aligned}
\tilde{H}\left[n_{p}, k_{p}\right] & =Y\left[n_{p}, k_{p}\right] / X\left[n_{p}, k_{p}\right] \\
& =H\left[n_{p}, k_{p}\right]+\tilde{Z}\left[n_{p}, k_{p}\right]
\end{aligned}
$$

where $n_{p}$ and $k_{p}$ denote the time and subcarrier index of the pilot symbol, respectively, and $\tilde{Z}\left[n_{p}, k_{p}\right]$ denotes additive noise. Letting $X\left[n_{p}, k_{p}\right]=1$ without loss of generality, $\tilde{Z}\left[n_{p}, k_{p}\right]$ can be assumed to be a zero-mean complex AWGN with variance $\sigma_{Z}^{2}$. The channel is also assumed to be bandlimited to a maximum Doppler frequency $f_{d}$ with a maximum time delay of $\tau_{\max }$. Then, when $d_{t} f_{d} T_{s} \leq 1 / 2$ and $d_{f} \tau_{\max } \Delta f_{c} \leq 1 / 2$, the CIR during the data symbol can be estimated by interpolating the CIRs at the instant of adjacent pilot symbols as [20]

$$
H[n, k]=\sum_{p=-\infty}^{\infty} \sum_{q=-\infty}^{\infty} H_{s}[n+p, k+q] w_{i d}[p, q]
$$

where $H_{s}[n, k]$ is equal to $H[n, k]$ at the pilot symbol (i.e., when $n=n_{p}$ and $k=k_{p}$ ), and zero, otherwise, and $w_{i d}[p, q]$ is the impulse response of an ideal two-dim non-causal brickwall type interpolator represented as

$$
w_{i d}[p, q]=\frac{\sin \left(\pi p / d_{t}\right) \sin \left(\pi q / d_{f}\right)}{\left(\pi p / d_{t}\right)\left(\pi q / d_{f}\right)} .
$$

Since $w_{i d}[p, q]$ is impractical for realization, it can be replaced by a non-ideal interpolator as

$$
\hat{H}[n, k]=\sum_{p=-\infty}^{\infty} \sum_{q=-\infty}^{\infty} \tilde{H}_{s}[n+p, k+q] w[p, q]
$$

where $w[p, q]$ is the coefficient of the interpolator and $\tilde{H}_{s}[n, k]$ is equal to $\tilde{H}[n, k]$ at the instant of pilot symbol transmission and zero, otherwise.

To obtain the CIR at the data symbol, the CIR estimated from the pilot symbol is first linearly interpolated in the frequency domain and then in the time domain as

$$
\begin{aligned}
\tilde{H}\left[n_{p}, k_{p}+k\right] & =\frac{k}{d_{f}}\left(\tilde{H}\left[n_{p}, k_{p}+d_{f}\right]-\tilde{H}\left[n_{p}, k_{p}\right]\right) \\
& +\tilde{H}\left[n_{p}, k_{p}\right], \quad 0<k<d_{f} \\
\tilde{H}\left[n_{p}+n, k\right] & =\frac{n}{d_{t}}\left(\tilde{H}\left[n_{p}+d_{t}, k\right]-\tilde{H}\left[n_{p}, k\right]\right) \\
& +\tilde{H}\left[n_{p}, k\right], \quad 0<n<d_{t} .
\end{aligned}
$$

Then, the estimated CIR is further processed using a two-dim MA FIR filter as

$\hat{H}[n, k]=\frac{1}{N_{t} N_{f}} \sum_{m_{1}=-M_{t}}^{M_{t}} \sum_{m_{2}=-M_{f}}^{M_{f}} \tilde{H}\left[n+m_{1} d_{t}, k+m_{2} d_{f}\right]$

where $N_{t}\left(=2 M_{t}+1\right)$ and $N_{f}\left(=2 M_{f}+1\right)$ denote the number of taps in the time and frequency domain, respectively. It can be shown from (18) that

$$
\begin{aligned}
& \hat{H}[n, k]=\sum_{p=-\infty}^{\infty} \sum_{q=-\infty}^{\infty} H_{s}[n+p, k+q] w[p, q] \\
& +\sum_{p=-\infty}^{\infty} \sum_{q=-\infty}^{\infty} Z_{s}[n+p, k+q] w[p, q] \\
& =\sum_{p=-\infty}^{\infty} \sum_{q=-\infty}^{\infty} H_{s}[n+p, k+q] w_{i d}[p, q] \\
& +\sum_{p=-\infty}^{\infty} \sum_{q=-\infty}^{\infty} H_{s}[n+p, k+q]\left(w[p, q]-w_{i d}[p, q]\right) \\
& +\sum_{p=-\infty}^{\infty} \sum_{q=-\infty}^{\infty} Z_{s}[n+p, k+q] w[p, q] \\
& =H[n, k]+\sum_{p=-\infty}^{\infty} \sum_{q=-\infty}^{\infty} H_{s}[n+p, k+q]\left(w[p, q]-w_{i d}[p, q]\right) \\
& +\sum_{p=-\infty}^{\infty} \sum_{q=-\infty}^{\infty} Z_{s}[n+p, k+q] w[p, q]
\end{aligned}
$$

where $Z_{s}[n, k]$ is equal to $\tilde{Z}[n, k]$ at the instant of pilot symbol transmission and zero, otherwise. Note that the first term of (23) is the desired CIR, the second term is the selfdistortion due to the use of a non-ideal interpolator, and the 
third term is the interference due to the background noise and the interference from other cells.

The MSE of the CIR estimate can be represented using Parseval's theorem as

$$
\begin{aligned}
\sigma_{e}^{2} & =E\left\{|\hat{H}[n, k]-H[n, k]|^{2}\right\} \\
& =\frac{1}{(2 \pi)^{2}} \int_{-\pi}^{\pi} \int_{-\pi}^{\pi} S_{H_{s}}\left(w_{1}, w_{2}\right)\left|W_{e}\left(w_{1}, w_{2}\right)\right|^{2} d w_{1} d w_{2} \\
& +\frac{\sigma_{Z}^{2}}{(2 \pi)^{2}\left(d_{t} d_{f}\right)} \int_{-\pi}^{\pi} \int_{-\pi}^{\pi}\left|W\left(w_{1}, w_{2}\right)\right|^{2} d w_{1} d w_{2} \\
& =\sigma_{S}^{2}+\sigma_{I}^{2}
\end{aligned}
$$

where $\sigma_{S}^{2}$ and $\sigma_{I}^{2}$ are respectively the MSE due to the selfdistortion and interference, and $S_{H_{s}}\left(w_{1}, w_{2}\right)$ is a sampled version of $S_{H}\left(w_{1}, w_{2}\right)$ represented as [20]

$S_{H_{s}}\left(w_{1}, w_{2}\right)=\frac{1}{\left(d_{t} d_{f}\right)^{2}} \sum_{n=0}^{d_{t}-1} \sum_{k=0}^{d_{f}-1} S_{H}\left(w_{1}-\frac{2 \pi}{d_{t}} n, w_{2}-\frac{2 \pi}{d_{f}} k\right)$.

Defining the interpolation error coefficient due to non-ideal interpolation by

$$
w_{e}[p, q]=w[p, q]-w_{i d}[p, q],
$$

the DFT of $w_{e}[p, q]$ can be represented as

$$
\begin{aligned}
& W_{e}\left(w_{1}, w_{2}\right) \\
& = \begin{cases}W\left(w_{1}, w_{2}\right)-d_{t} d_{f}, & \left|w_{1}\right| \leq \pi / d_{t} \text { and }\left|w_{2}\right| \leq \pi / d_{f} \\
W\left(w_{1}, w_{2}\right), & \text { otherwise }\end{cases}
\end{aligned}
$$

where $W\left(w_{1}, w_{2}\right)$ is the two-dim DFT of $w[p, q]$. For example, $W\left(w_{1}, w_{2}\right)$ of the cascaded CEF can be represented as

$$
\begin{aligned}
W\left(w_{1}, w_{2}\right) & =\frac{1}{d_{t} d_{f}}\left(\frac{\sin \left(w_{1} d_{t} / 2\right) \sin \left(w_{2} d_{f} / 2\right)}{\sin \left(w_{1} / 2\right) \sin \left(w_{2} / 2\right)}\right)^{2} \\
& \times \frac{1}{N_{t} N_{f}} \frac{\sin \left(w_{1} d_{t} N_{t} / 2\right) \sin \left(w_{2} d_{f} N_{f} / 2\right)}{\sin \left(w_{1} d_{t} / 2\right) \sin \left(w_{2} d_{f} / 2\right)} .
\end{aligned}
$$

Neglecting the MSE in the out-of-passband spectrum (i.e., $w_{1} \notin\left[-\pi / d_{t} \pi / d_{t}\right]$ for and $\left.w_{2} \notin\left[-\pi / d_{f} \pi / d_{f}\right]\right)$ and using a Taylor series approximation of $\left|W_{e}\left(w_{1}, w_{2}\right)\right|^{2}$, we can approximate the MSE in (24) as

$$
\begin{aligned}
\sigma_{e}^{2} & \approx \frac{1}{\left(d_{t} d_{f}\right)^{2}}\left(c_{I F, 0} \bar{w}_{1}^{(2)} \bar{w}_{2}^{(2)}+c_{I F, 1} \bar{w}_{1}^{(4)}+c_{I F, 2} \bar{w}_{2}^{(4)}\right) \\
& +\frac{1}{N_{t} N_{f} \bar{\gamma}}
\end{aligned}
$$

where $\bar{w}_{1}^{(k)}$ is the $k$-th order moment of the Doppler spectrum in (13), $\bar{w}_{2}^{(k)}$ is the $k$-th order moment of the power-delay profile represented as

$$
\bar{w}_{2}^{(k)}=\frac{1}{2 \pi} \int_{-\pi}^{\pi} w^{n} S_{H_{2}}(w) d w
$$

and $c_{I F, 0}, c_{I F, 1}$ and $c_{I F, 2}$ are the coefficients of Taylor series approximation of $\left|W_{e}\left(w_{1}, w_{2}\right)\right|^{2}$

$$
\begin{aligned}
c_{I F, 0} & \approx d_{t}^{2} d_{f}^{2}\left(d_{t}^{2} N_{t}^{2}\right)\left(d_{f}^{2} N_{f}^{2}\right) / 288 \\
c_{I F, 1} & \approx d_{t}^{2} d_{f}^{2}\left(d_{t}^{2} N_{t}^{2}\right)^{2} / 576 \\
c_{I F, 2} & \approx d_{t}^{2} d_{f}^{2}\left(d_{f}^{2} N_{f}^{2}\right)^{2} / 576
\end{aligned}
$$

for $N_{t}, N_{f} \gg 1$. Here, the use of a fourth-order Taylor approximation is sufficient since the effect of higher order terms is negligible. Then, it can be shown that

$$
\begin{aligned}
\sigma_{e}^{2} & \approx \frac{1}{576}\left(2 \bar{w}_{1}^{(2)} \bar{w}_{2}^{(2)} d_{t}^{2} d_{f}^{2} N_{t}^{2} N_{f}^{2}+\bar{w}_{1}^{(4)} d_{t}^{4} N_{t}^{4}\right. \\
& \left.+\bar{w}_{2}^{(4)} d_{f}^{4} N_{f}^{4}\right)+\frac{1}{N_{t} N_{f} \bar{\gamma}} \\
& \geq \frac{\left(d_{t} d_{f} N_{t} N_{f}\right)^{2}}{288}\left(\bar{w}_{1}^{(2)} \bar{w}_{2}^{(2)}+\sqrt{\bar{w}_{1}^{(4)} \bar{w}_{2}^{(4)}}\right)+\frac{1}{N_{t} N_{f} \bar{\gamma}}
\end{aligned}
$$

where the equality holds when

$$
\bar{w}_{1}^{(4)} d_{t}^{4} N_{t}^{4}=\bar{w}_{2}^{(4)} d_{f}^{4} N_{f}^{4} .
$$

Assuming that $N_{t}$ and $N_{f}$ are continuous variables for ease of analysis, the optimum $N_{t} N_{f}$ can uniquely be determined by solving

$$
\frac{\partial \sigma_{e}^{2}}{\partial\left(N_{t} N_{f}\right)}=0
$$

Thus, it can be shown that the minimum MSE is

$$
\begin{aligned}
& \sigma_{e, \min }^{2}=\frac{1.5}{\left(N_{t} N_{f}\right)_{o p t} \bar{\gamma}} \\
& =(3 / 128)^{1 / 3}\left(d_{t} d_{f}\right)^{2 / 3}(\bar{\gamma})^{-2 / 3}\left(\bar{w}_{1}^{(2)} \bar{w}_{2}^{(2)}+\sqrt{\bar{w}_{1}^{(4)} \bar{w}_{2}^{(4)}}\right)^{1 / 3}
\end{aligned}
$$

where

$$
\left(N_{t} N_{f}\right)_{o p t}=\frac{1}{\left(d_{t} d_{f}\right)^{2 / 3}}\left(\frac{144 \bar{\gamma}^{-1}}{\bar{w}_{1}^{(2)} \bar{w}_{2}^{(2)}+\sqrt{\bar{w}_{1}^{(4)} \bar{w}_{2}^{(4)}}}\right)^{1 / 3} .
$$

The minimum MSE $\sigma_{e, \min }^{2}$ increases as the pilot spacing, the interference power or/and the moments of the channel spectrum increase. Finally, the optimum tap size $\hat{N}_{t}$ and $\hat{N}_{f}$ can be determined by

$$
\begin{aligned}
& \hat{N}_{t}=\frac{\left(d_{t} d_{f}\right)^{1 / 6}}{d_{t}}\left(\frac{\bar{w}_{2}^{(4)}}{\bar{w}_{1}^{(4)}}\right)^{1 / 8}\left(\frac{144 \bar{\gamma}^{-1}}{\bar{w}_{1}^{(2)} \bar{w}_{2}^{(2)}+\sqrt{\bar{w}_{1}^{(4)} \bar{w}_{2}^{(4)}}}\right)^{1 / 6} \\
& \hat{N}_{f}=\frac{\left(d_{t} d_{f}\right)^{1 / 6}}{d_{f}}\left(\frac{\bar{w}_{2}^{(4)}}{\bar{w}_{1}^{(4)}}\right)^{-1 / 8}\left(\frac{144 \bar{\gamma}^{-1}}{\bar{w}_{1}^{(2)} \bar{w}_{2}^{(2)}+\sqrt{\bar{w}_{1}^{(4)} \bar{w}_{2}^{(4)}}}\right)^{1 / 6} .
\end{aligned}
$$

Thus, the two-dim MA FIR CEF should be optimized according to the pilot spacing, the average SIR, and the second and fourth order moment of the Doppler spectrum and powerdelay profile of the channel. Note that this information can be predetermined or obtained using the proposed CSI estimator. As the pilot spacing and/or the average SIR increase, the required tap size of the two-dim MA FIR CEF decreases. As the moments of $w_{1}$ or $w_{2}$ decrease (i.e., slowly varying or less frequency selective channel), the required tap size increases, 
and vice versa. Although the analytic result (37) is obtained assuming the cascading use of linear interpolation and MA FIR filters as the CEF, the same analytic method can easily be applied to the use of other CEFs by simply replacing $W\left(w_{1}, w_{2}\right)$ in (28).

We have considered the use of real-coefficient CEFs to reduce the computational complexity instead of complexcoefficient CEFs. However, it may experience performance degradation when the channel has not a symmetric spectrum (e.g., the power-delay profile). That is, the use of a realcoefficient filter may require a larger bandwidth to properly preserve the signal as illustrated in Fig. 2 (a). Without the use of a complex-coefficient CEF, this problem can nicely be handled with the use of a real-coefficient CEF by shifting the power-delay profile as illustrated in Fig. 2 (b). Thus, realcoefficient CEFs can be employed without suffering performance degradation due to excessive noise.

The delay-shifting by an amount of $\tau_{s h}$ can easily be achieved by rotating the phase of the received symbol by $\exp \left(j k 2 \pi \tau_{s h} / T_{s}\right)$ for the $k$-th subcarrier, $k=0,1, \ldots$, $K-1$. A similar idea was considered in [22], where the delay is only shifted by a fixed amount of one-half CP duration. Thus, it cannot provide optimum performance in the presence of various power-delay profiles. This problem can be alleviated by determining the amount of delay-shift according to the distribution of the power-delay profile. To this end, the optimum amount of delay-shift is analytically determined to minimize the MSE of the CIR estimate.

The MSE of the channel estimate with phase-rotation by $w_{s h}\left(=2 \pi \tau_{s h} / T_{s}\right)$ can be represented as

$$
\begin{aligned}
& \sigma_{e}^{2}\left(w_{s h}\right) \\
& =\frac{1}{(2 \pi)^{2}} \int_{-\pi}^{\pi} \int_{-\pi}^{\pi} S_{H_{s}}\left(w_{1}, w_{2}+w_{s h}\right)\left|W_{e}\left(w_{1}, w_{2}\right)\right|^{2} d w_{1} d w_{2} \\
& \approx \frac{1}{(2 \pi)^{2}} \int_{-\pi}^{\pi} \int_{-\pi}^{\pi} S_{H_{1}}\left(w_{1}\right) S_{H_{2}}\left(w_{2}\right)\left(c_{I F, 0} w_{1}^{2}\left(w_{2}-w_{s h}\right)^{2}\right. \\
& \left.+c_{I F, 1} w_{1}^{4}+c_{I F, 2}\left(w_{2}-w_{s h}\right)^{4}\right) d w_{1} d w_{2} .
\end{aligned}
$$

For a given CEF (i.e., $c_{I F, 0}, c_{I F, 1}$ and $c_{I F, 2}$ ), the optimum phase-rotation $\hat{w}_{s h}$ minimizing the MSE can be obtained by

$$
\begin{aligned}
\left.\frac{\partial \sigma_{s}^{2}\left(w_{s h}\right)}{\partial w_{s h}}\right|_{w_{s h}=\hat{w}_{s h}} & =-\frac{2 c_{I F, 0} \bar{w}_{1}^{(2)}}{2 \pi} \sum_{l=0}^{L-1} \sigma_{l}^{2}\left(w_{l}-\hat{w}_{s h}\right) \\
& -\frac{4 c_{I F, 2}}{2 \pi} \sum_{l=0}^{L-1} \sigma_{l}^{2}\left(w_{l}-\hat{w}_{s h}\right)^{3} \\
& =0 .
\end{aligned}
$$

Thus, the optimum $\hat{w}_{s h}$ can be determined by

$$
\begin{aligned}
& c_{I F, 0} \bar{w}_{1}^{(2)}\left(\bar{w}_{2}^{(1)}-\hat{w}_{s h}\right) \\
& +2 c_{I F, 2}\left(\bar{w}_{2}^{(3)}-3 \bar{w}_{2}^{(2)} \hat{w}_{s h}+3 \bar{w}_{2}^{(1)} \hat{w}_{s h}^{2}-\hat{w}_{s h}^{3}\right)=0 .
\end{aligned}
$$

We can calculate $\hat{w}_{s h}$ by numerically solving a third-order polynomial equation, which may not be easily realizable. Instead, we consider the use of a suboptimum solution easily

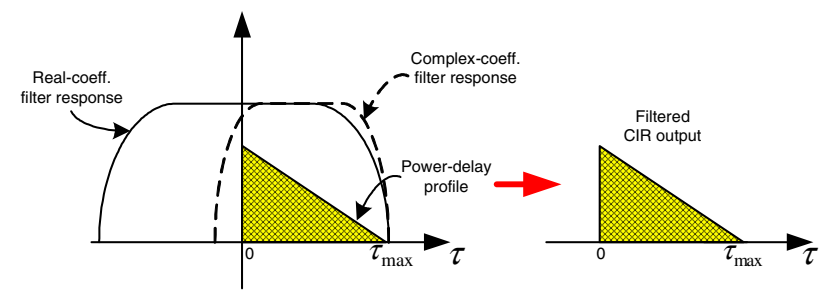

(a) Conventional scheme

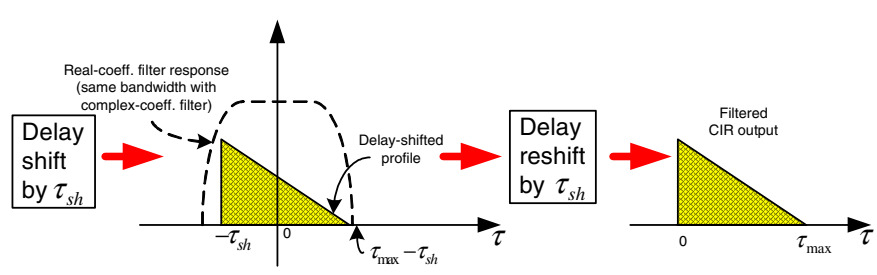

(b) Proposed scheme

Fig. 2. The proposed real coefficient CEF with delay-shifting.

realizable in practice. Neglecting the second term in (40), the suboptimum $\hat{w}_{s h}^{\prime}$ is equal to the first order moment of the power-delay profile (i.e., the mean delay spread) as

$$
\hat{w}_{s h}^{\prime}=\bar{w}_{2}^{(1)}=\sum_{l=0}^{L-1} \sigma_{l}^{2} w_{l} .
$$

Thus, the delay-shift processing can be realized using the firstorder moment of the power-delay profile as shown in Fig. 1.

\section{C. $A M C$}

The performance of an OFDM system can substantially vary according to the channel diversity. As an example, Fig. 3 depicts the PER performance of the OFDM system in consideration, whose parameters are summarized in Table 1 [18]. When the channel has an rms delay spread $\left(\tau_{r m s}\right)$ of $10 \mathrm{nsec}$ and a maximum Doppler frequency $f_{d}$ of $16 \mathrm{~Hz}$, it can be seen that 64-QAM can be employed when the SIR is higher than $21.0 \mathrm{~dB}$, and 16-QAM and QPSK can be employed when the SIR is higher than $17.0 \mathrm{~dB}$ and $13.0 \mathrm{~dB}$, respectively, for PER $\leq 10^{-2}$. Otherwise, BPSK modulation should be employed. On the other hand, the SIR threshold values at $\mathrm{PER}=10^{-2}$ are significantly reduced when $\tau_{r m s}=436 \mathrm{nsec}$ and $f_{d}=1343$ $\mathrm{Hz}$. The difference is mainly due to the fact that the data signal in each transmission block experiences different degrees of diversity in the time and frequency domain, resulting in substantially different PER curves. Table 2 summarizes the optimum SIR threshold in different channel environments. It can be seen that the threshold decreases as the CIR varies faster or becomes frequency selective. This implies that the performance can be improved if the SIR threshold for the AMC is properly adjusted according to the channel condition.

In this paper, we consider the selection of the AMC mode by adjusting the threshold based on the rms value of the Doppler 
TABLE I

SYSTEM PARAMETERS

\begin{tabular}{|c|c|}
\hline Parameters & Values \\
\hline Duplexing & FDD \\
\hline Carrier frequency & $5.8 \mathrm{GHz}$ \\
\hline Bandwidth & $100 \mathrm{MHz}$ \\
\hline Symbol duration & $20.48 \mu \mathrm{sec}$ \\
\hline Guard interval & $5 \mu \mathrm{sec}$ \\
\hline Number of subcarriers & 2048 \\
\hline Channel coding & Zig-zag code \\
\hline Number of symbols in data block & 8/128 (time/frequency) \\
\hline Pilot interval $\left(d_{t}, d_{f}\right)$ & $(8,4)$ \\
\hline Channel & Rayleigh (Jakes spectrum) \\
\hline Power-delay profile & Exponential (delay spacing: $10 \mathrm{nsec}$ ) \\
\hline Hopping and interleaving & $\begin{array}{l}\text { Equi-distant frequency hopping } \\
\text { and block interleaving }\end{array}$ \\
\hline HARQ & $\begin{array}{c}\text { Incremental redundancy } \\
\text { (up to } 3 \text { retrans., initial code rate: } 3 / 4 \text { ) }\end{array}$ \\
\hline Correlation period & 208.73 msec (8192 symbol duration) \\
\hline $\begin{array}{l}\text { Number of correlators } \\
\text { in CSI estimator }(W)\end{array}$ & 3 \\
\hline
\end{tabular}

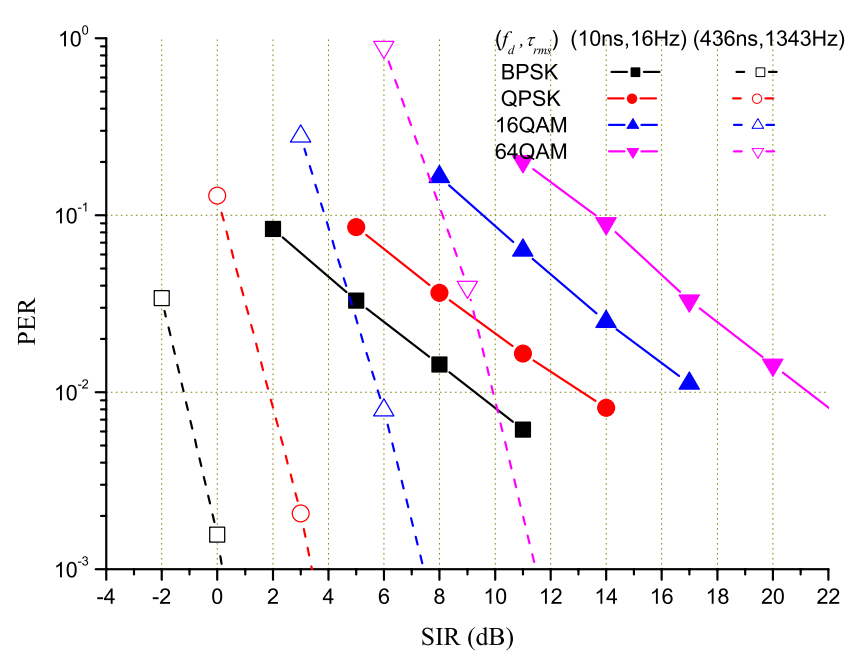

Fig. 3. PER performance with perfect CIR.

spectrum and power-delay profile as

$$
\begin{aligned}
f_{r m s} & =\frac{1}{2 \pi T_{s}} \sqrt{\bar{w}_{1}^{(2)}-\left(\bar{w}_{1}^{(1)}\right)^{2}} \\
\tau_{r m s} & =\frac{1}{2 \pi \Delta f_{c}} \sqrt{\bar{w}_{2}^{(2)}-\left(\bar{w}_{2}^{(1)}\right)^{2}} .
\end{aligned}
$$

These two parameters, which can be calculated using the firstand second-order moment estimated by the proposed CSI estimator, indicate how much the channel is varying in the time and frequency domain, respectively. In practice, the SIR threshold for a certain channel condition can be calculated by interpolating the nearest two threshold values in Table 2 . For the purpose of interpolation, the use of a simple two-dim linear interpolator may be sufficient.
TABLE II

SIR THRESHOLD FOR THE MODE SELECTION AT A PER OF $10^{-2}(d B)$

\begin{tabular}{|c|c|c|c|}
\hline$f_{d}(\mathrm{~Hz})$ & $\begin{array}{c}\text { 64QAM-16QAM } \\
\left(\tau_{r m s}=10 / 167 / 436 \mathrm{~ns}\right)\end{array}$ & $\begin{array}{c}\text { 16QAM-QPSK } \\
(10 / 167 / 436 \mathrm{~ns})\end{array}$ & $\begin{array}{c}\text { QPSK-BPSK } \\
(10 / 167 / 436 \mathrm{~ns})\end{array}$ \\
\hline 16 & $21.0 / 16.5 / 14.7$ & $17.0 / 12.5 / 10.7$ & $13.0 / 8.1 / 5.2$ \\
\hline 228 & $14.0 / 12.7 / 10.3$ & $11.0 / 8.7 / 6.0$ & $6.2 / 4.1 / 2.2$ \\
\hline 950 & $12.5 / 11.3 / 10.0$ & $8.8 / 7.1 / 5.7$ & $3.2 / 2.7 / 1.8$ \\
\hline
\end{tabular}

\section{Performance Evaluation}

To verify the performance of the proposed schemes, computer simulation is performed with system parameters summarized in Table 1 [18]. To obtain the time diversity and mitigate the effect of SIR estimation inaccuracy, we consider the use of a hybrid ARQ with an incremental redundancy type (HARQIR), where retransmission is maximally allowed three times with an initial code rate of $3 / 4$. The pilot signal is transmitted with $d_{t}=8$ and $d_{f}=4$ to support the presumed worst channel condition with $f_{d}=1343 \mathrm{~Hz}(250 \mathrm{~km} / \mathrm{h})$ and $\tau_{r m s}=436 \mathrm{nsec}$ [21]. The CSI estimator employs three correlators $(W=3)$ to measure the first-, second- and fourth-order moment as well as the average SIR.

Fig. 4 depicts the MSE performance of the proposed CIR estimation scheme. For performance comparison, we consider the use of a two-dim Wiener CEF having 35 and 9 taps in the time and frequency domain, respectively, since the use of additional taps provides negligible performance improvement. We also consider the use of two conventional CIR estimation schemes; a linear interpolation scheme and an adaptive FIR filter selection scheme in [17]. It can be seen that the use of the proposed CEF scheme provides performance slightly worse than the use of Wiener CEF. This performance inferiority at high SIR is partly due to the use of a smaller tap size than the Wiener filter. It can be seen that the scheme in 
TABLE III

COMPARISON OF COMPUTATIONAL COMPLEXITY

\begin{tabular}{|c|c|c|}
\hline Type & $\begin{array}{c}\text { Multiplications/symbol } \\
(\text { example*) }\end{array}$ & $\begin{array}{c}\text { Matrix inversion } \\
(\text { example*) }\end{array}$ \\
\hline 2-D Wiener & $4 N_{t} N_{f}(1260)$ & $N_{t} N_{f} \times N_{t} N_{f}(315 \times 315)$ \\
\hline Two & $4\left(N_{f} / d_{t}+N_{t}\right)(144.5)$ & $\begin{array}{c}N_{t} \times N_{t} \text { and } N_{f} \times N_{f} \\
(35 \times 35 \text { and } 9 \times 9)\end{array}$ \\
1-D Wiener & & None $(0)$ \\
\hline Proposed & $2\left(d_{f}^{2}-1\right) /\left(d_{t} d_{f}\right)+2(2.93)$ & \\
\hline
\end{tabular}

*. When $N_{t}=35, N_{f}=9, d_{t}=8$ and $d_{f}=4$.

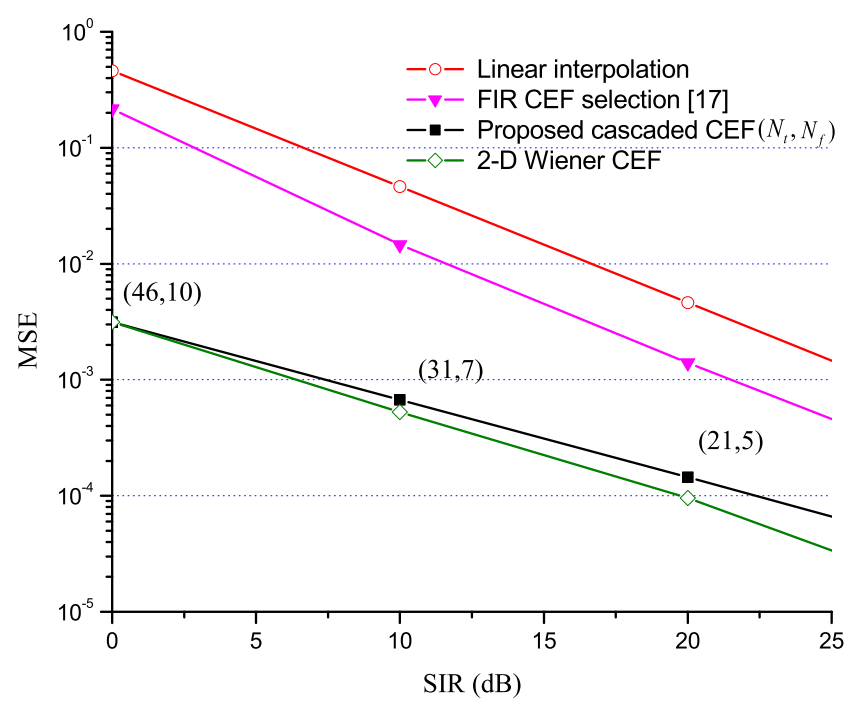

Fig. 4. MSE performance when $\tau_{r m s}=10 \mathrm{nsec}$ and $f_{d}=16 \mathrm{~Hz}$.

[17] does not provide good performance since the CEF is heuristically selected. As another measure, the computational complexity is compared in Table 3. Here, we only consider the computational complexity for the CEF assuming that all the schemes require the same complexity for the CSI estimation. It can be seen that the proposed scheme can be realized with significantly reduced complexity.

Fig. 5 depicts the MSE performance as a function of the rms delay spread (i.e., different path exponents) when the proposed cascaded CEF scheme is employed with the proposed delayshifting process. It can be seen that the proposed scheme is quite effective particularly when the rms delay is large and that the suboptimum scheme provides near optimum performance.

Fig. 6 depicts the throughput performance of the proposed AMC for some values of $\tau_{r m s}$ and $f_{d}$ with the use of perfect CIR and CSI. Here, 'Optimum Th' denotes the case when the optimum SIR threshold is employed, while 'Fixed Th' and 'Adaptive Th' indicate the case when the fixed threshold and the proposed adaptive threshold are used, respectively. The throughput is measured by counting the data bits in the blocks transmitted without any error. The fixed threshold scheme optimally determines the threshold assuming that the channel within a block is invariant in the time and frequency domain. It can be seen that the proposed scheme can provide near optimum performance, while the conventional scheme yields significant performance degradation. This performance

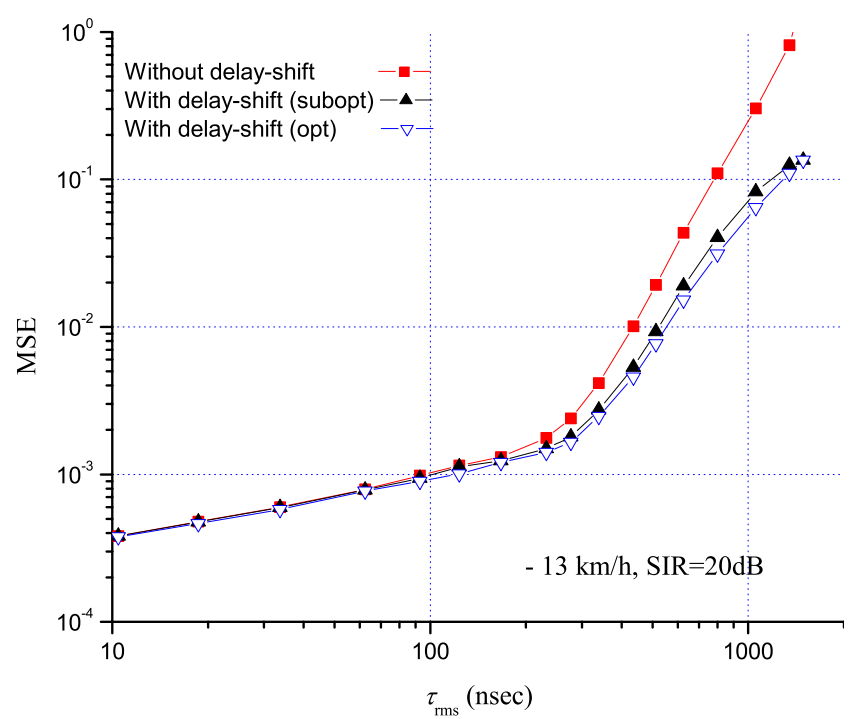

Fig. 5. MSE of CIR estimate with and without the delay-shifting.

differentiation increases as the delay and/or Doppler spread increase.

The overall throughput performance of the proposed OFDM transceiver is evaluated in Fig. 7 when all the proposed schemes in Fig. 1 are employed. The 'Ideal' case assumes the use of perfect measurement of the CIR and CSI as well as the optimum SIR threshold. The 'Conventional' scheme employs a linear interpolator as the CEF without delay-shifting for the CIR estimation and uses a fixed SIR threshold. The 'Proposed' scheme employs the proposed adaptive CIR estimator and AMC scheme whose parameters are accessible by the proposed CSI estimator. To evaluate the performance of the CSI estimator, we evaluate the performance of the proposed scheme under two conditions; perfect and estimated CSI. It can be seen that the proposed scheme significantly improves the performance over the conventional one in a wide range of channel condition. When the channel has a small diversity (i.e., low $\tau_{r m s}$ and small $f_{d}$ ), the performance can significantly be degraded by excessive noise due to the use of a fixed CEF. When the channel has a large diversity, the performance of the conventional scheme is also significantly degraded due to the use of an inappropriate SIR threshold. It can be seen that the proposed CSI estimator can provide sufficient estimation accuracy, yielding throughput performance close to the use of perfect CSI. 


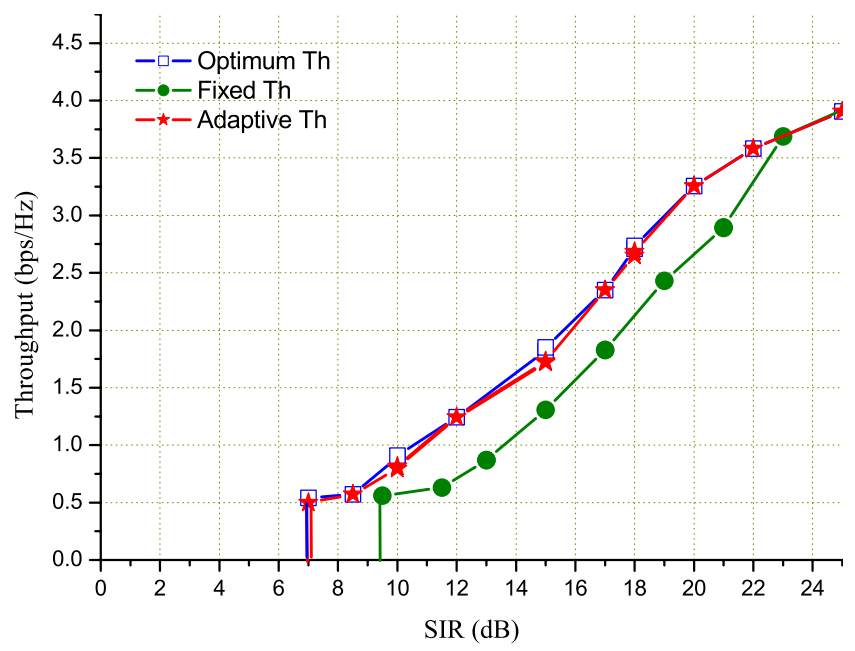

(a) When $\tau_{r m s}=41 \mathrm{nsec}$ and $f_{d}=57 \mathrm{~Hz}(10 \mathrm{~km} / \mathrm{h})$

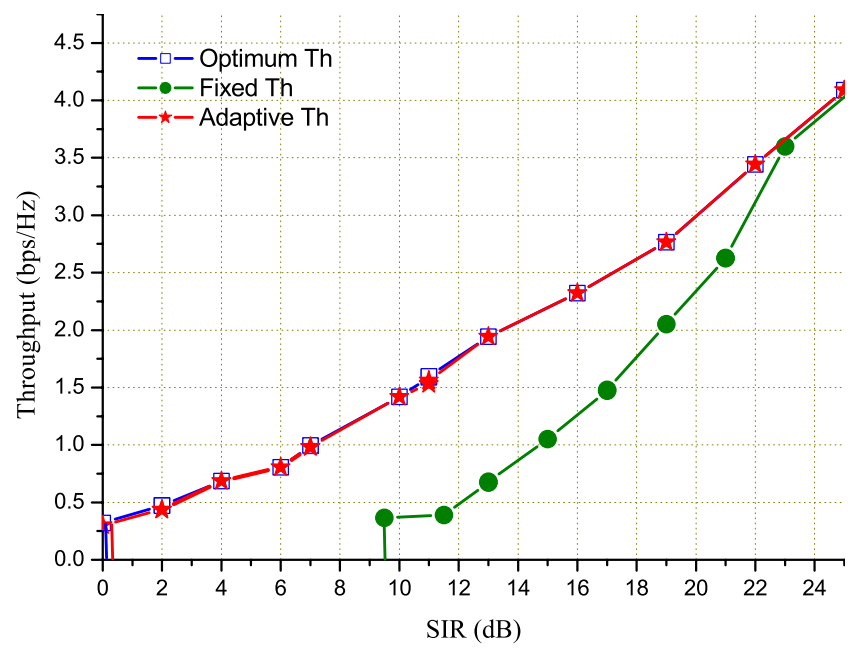

(b) When $\tau_{r m s}=270 \mathrm{nsec}$ and $f_{d}=644 \mathrm{~Hz}(120 \mathrm{~km} / \mathrm{h})$

Fig. 6. Throughput performance with perfect CIR and CSI.

\section{CONCLUSIONS}

In this paper, we have considered the use of channel-aware techniques for the design of an OFDM wireless transceiver. For better design of a channel-aware OFDM transceiver, we considered the use of low order moments of the Doppler spectrum and power-delay profile as well as the average SIR as the CSI. We have first designed a single unified scheme that can estimate these CSI parameters altogether. Then, we have devised a CIR estimator that employs an adaptive CEF with real coefficients. Finally, we have improved link-level performance by adjusting the AMC threshold according to the estimated CSI. The performance of the proposed scheme is verified by computer simulation. The simulation results show that the proposed scheme can provide near optimum performance in a wide variation of channel condition.

\section{REFERENCES}

[1] ETSI, "Digital video broadcasting: framing, channel coding and modulation for digital terrestrial television," Aug. 1997.

[2] ISO/IEC, "IEEE Std 802.11a Part 11 Amendment 1: High-speed physical layer in the $5 \mathrm{GHz}$ band, 1999 .

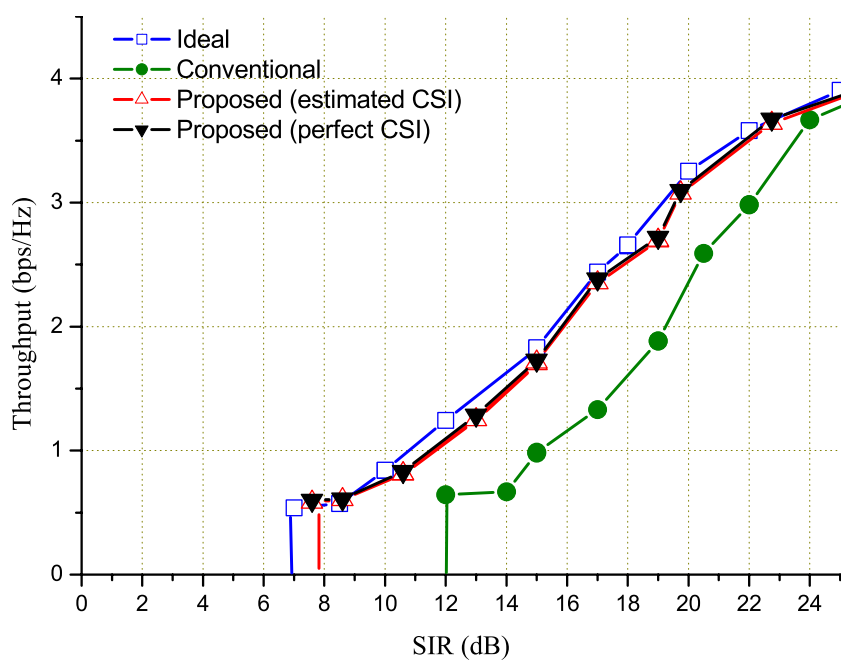

(a) When $\tau_{r m s}=41 \mathrm{nsec}$ and $f_{d}=57 \mathrm{~Hz}(10 \mathrm{~km} / \mathrm{h})$

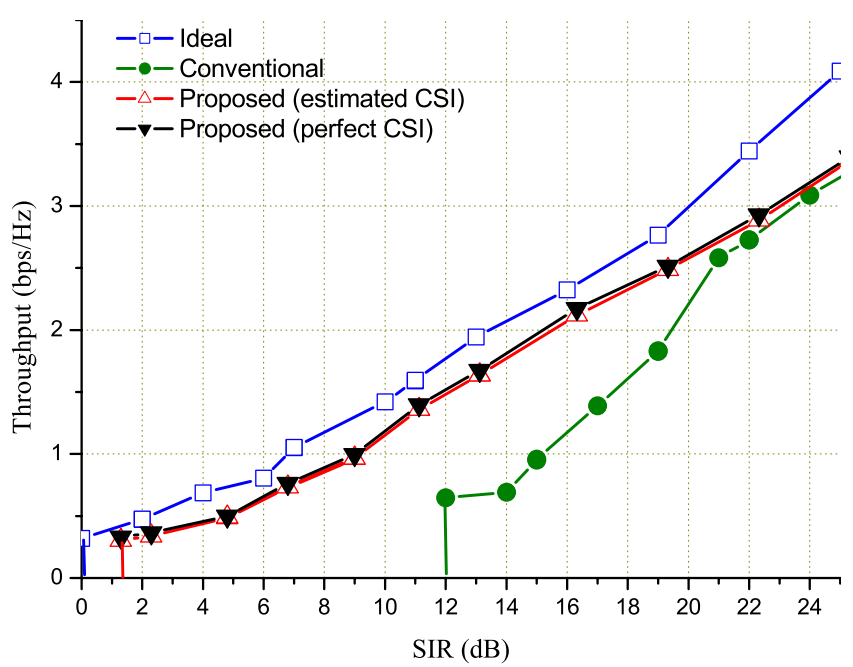

(b) When $\tau_{r m s}=270 \mathrm{nsec}$ and $f_{d}=644 \mathrm{~Hz}(120 \mathrm{~km} / \mathrm{h})$

Fig. 7. Throughput performance of the proposed scheme.

[3] H. Atarashi, S. Abeta, and M. Sawahashi, "Performance evaluation of coherent high-speed TD-OFCDM broadband packet wireless access in forward link employing multi-level modulation and hybrid ARQ," IEICE Trans. Commun., vol. E84-B, no. 7, pp. 1670-1680, July 2001.

[4] H. Sampath, S. Talwar, J. Tellado, V. Erceg, and A. Paulraj, "A fourthgeneration MIMO-OFDM broadband wireless system: design, performance, and field trial results," IEEE Commun. Mag., vol. 40, no. 9, pp. 143-149, Sept. 2002.

[5] J. Cavers, "Variable-rate transmission for Rayleigh fading channels," IEEE Trans. Commun., vol. 20, no. 1, pp. 15-22, Feb. 1972.

[6] Q. Xiaoxin and K. Chawla, "On the performance of adaptive modulation in cellular systems," IEEE Trans. Commun., vol. 47, no. 6, pp. 884-895, June 1999.

[7] C. Tepedelenlioglu and G. B. Giannakis, "On velocity estimation and correlation properties of narrow-band mobile communication channels," IEEE Trans. Veh. Commun., vol. 50, no. 4, pp. 1039-1052, July 2001.

[8] J.-W. Choi and Y.-H. Lee, "Efficient channel estimation in DS-CDMA systems," IEICE Trans. Commun., vol. E87-B, no. 6, pp. 1470-1478, June 2004.

[9] E. Cianca, A. D. Luise, M. Ruggieri, and R. Prasad, "Channel-adaptive techniques in wireless communications: an overview," Wireless Commun. and Mobile Comput., vol. 2, no. 8, pp. 799-813, 2002.

[10] J.-W. Choi and Y.-H. Lee, "Design of 2-D channel estimation filters for OFDM systems," in Proc. IEEE ICC'05, pp. 2568-2572.

[11] S. Catreux, V. Erceg, D. Gesbert, and R. W. Heath Jr., "Adaptive modulation and MIMO coding for broadband wireless data networks," IEEE Commun. Mag., vol. 40, no. 6, pp. 108-115, June 2002. 
[12] Y. Li, L. J. Cimini Jr., and N. R. Sollenberger, "Robust channel estimation for OFDM systems with rapid dispersive fading channels," IEEE Trans. Coummun., vol. 46, no. 7, pp. 902-915, July 1998.

[13] P. Bello, "Some techniques for the instantaneous real-time measurement of multipath and Doppler spread," IEEE Trans. Commun., vol. 13, no. 3, pp. 285-292, Sept. 1965.

[14] W. C. Jakes, Microwave Mobile Communications. John Wiley and Sons, 1974.

[15] P. Hoeher, S. Kaiser, and P. Robertson, "Two-dimensional pilot-symbol aided channel estimation by Wiener filtering,' in Proc. IEEE ICASSP'97, pp. 1845-1848.

[16] S. Coleri, M. Ergen, A. Puri, and A. Bahai, "Channel estimation techniques based on pilot arrangement in OFDM systems," IEEE Trans. Broadcasting, vol. 48, no. 3, pp. 223-229, Sept. 2002.

[17] T. Onizawa, M. Mizoguchi, T. Sakata, and M. Morikura, "A simple adaptive channel estimation scheme for OFDM systems," in Proc. IEEE VTC'99 Fall, pp. 279-283.

[18] J. Moon, J.-Y. Ko, and Y.-H. Lee, "A framework design for nextgeneration radio access system," IEEE J. Sel. Areas Commun., vol. 24, no. 3, pp. 554-564, Mar. 2006.

[19] A. J. Goldsmith and P. P. Varaiya, "Capacity of fading channels with channel side information," IEEE Trans. Inf. Theory, vol. 43, no. 6, pp. 1986-1992, Nov. 1997.

[20] A. V. Oppenheim and R. W. Schafer, Discrete-Time Signal Processing, Second Edition. Prentice Hall, 1999.

[21] J.-W. Choi and Y.-H. Lee, "Optimum pilot pattern for channel estimation in OFDM systems," IEEE Trans. Wireless Commun., vol. 4, no. 5, pp. 2083-2088, Sept. 2005.

[22] B. Yang, Z. Cao, and K. B. Letaief, "Analysis of low-complexity windowed FT-based MMSE channel estimator for OFDM systems," IEEE Trans. Commun., vol. 49, no. 11, pp. 1977-1987, Nov. 2001.

[23] L. Hanzo, W. Webb, and T. Keller, Single- and Multi-Carrier Quadrature Amplitude Modulation: Principles and Applications for Personal Communications, WLANs and Broadcasting. John Wiley and Sons, 2000.

[24] J. M. Torrance and L. Hanzo, "Optimization of switching levels for adaptive modulation in slow Rayleigh fading," Electron. Lett., vol. 32, no. 13, pp. 1167-1169, June 1996.

[25] T. Keller and L. Hanzo, "Adaptive multicarrier modulation: a convenient framework for time-frequency processing in wireless communications," Proc. of IEEE, vol. 88, no. 5, pp. 611-640, May 2000.

[26] J. Lee, R. Arnott, K. Hamabe, and N. Takano, "Adaptive modulation switching level control in high speed downlink packet access transmission," in Proc. Int. Conf. 3G Mobile Commun. Tech.'02, pp.156-159.

[27] S. Muneta, Y. Matsumoto, N. Mochizuki, and M. Umehira, "A new frequency domain link adaptation scheme for broadband OFDM systems," in Proc. IEEE VTC'99 Fall, pp. 253-257.

[28] H. Harada, T. Yamamura, Y. Kamio, and M. Fujise, "Adaptive modulated OFDM radio transmission scheme using a new channel estimation method for future broadband mobile communication systems," IEICE Trans. Commun., vol. E85-B, no. 12, pp. 2785-2796, Dec. 2002.

[29] K. Balachandran, S. R. Kadaba, and S. Nanda, "Channel quality estimation and rate adaptation for cellular mobile radio," IEEE J. Sel. Areas Commun., vol. 17, no. 7, pp. 1244-1256, July 1999.

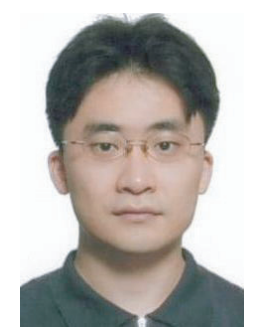

Ji-Woong Choi (S'00-M'04) received the B.S., M.S. and Ph.D. degree in electrical engineering from Seoul National University (SNU), Korea, in 1998, 2000 and 2004, respectively. From 2004 to 2005 , he worked as a postdoctoral research associate in Inter-University Semiconductor Center (ISRC), SNU, Korea. Since Nov. 2005, he has been with Department of Electrical Engineering, Stanford University, Stanford, CA, as a postdoctoral visiting scholar. He has also been serving as a Consultant to GCT Semiconductor, San Jose, CA, for development of handheld digital TV receivers since July 2006. His research areas are wireless transmission systems including OFDM and CDMA systems and signal processing for communication systems. He received Silver Award at Samsung Humantech Paper Contest in Feb. 2005.

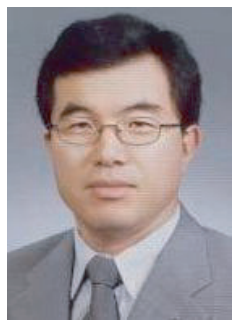

Yong-Hwan Lee (S'77-M'89) received his B.S. degree from Seoul National University (SNU), Korea, in 1977, M.S. degree from Korea Advanced Institute of Science and Technology (KAIST) in 1980 and Ph.D. degree from the University of Masachusetts, Amherst, U.S.A. in 1989, all in electrical engineering. From 1980 to 1985 , he was with the Korea Agency for Defense Development as a Senior Engineer. From 1989 to 1994 , he worked for Motorola, Inc., where he was involved in the development of data transmission systems including high-speed modems. Since 1994, he has been with the School of Electrical Engineering SNU, as a faculty member. He served as Director of the Institute of New Media and Communications in SNU from 2001 to 2003 and Vice Chair of the School of Electrical Engineering, SNU from 2003 to 2005 . He also served as a Guest Editor of international journals, including IEEE Journal on Selected Areas in Communications and IEEE Journal of Communications and Networks. He has been working on the design of wireline/wireless transceivers, including power-line modems, wideband CDMA and OFDMbased transceivers such as mobil WiMax and 4G. 